\title{
Size-consistent explicitly correlated triple excitation correction
}

\author{
Mihály Kállaya), Réka A. Horváth, László Gyevi-Nagy, and Péter R. Nagy ${ }^{1}$ \\ Department of Physical Chemistry and Materials Science, \\ Budapest University of Technology and Economics, H-1521 Budapest, P.O.Box 91, \\ Hungary
}

A new approach is proposed to reduce the basis set incompleteness error of the triple excitation correction in explicitly correlated coupled-cluster singles and doubles with perturbative triples $[\mathrm{CCSD}(\mathrm{T})]$ calculations. Our method is similar to the intuitive triples correction approach of Knizia and co-workers [J. Chem. Phys. 130, 054104 (2009)], but in contrast to the latter, is size-consistent. The new approximation is easy to implement, and its overhead is negligible with respect to the conventional (T) correction. The performance of the approach is assessed for atomization, reaction, and interaction energies as well as for bond lengths and harmonic vibrational frequencies. The advantages of its size-consistency are also demonstrated.

a) Electronic mail: kallay.mihaly@vbk.bme.hu 


\section{INTRODUCTION}

If accurate results are desired in computational chemistry, mean-field techniques, such as Hartree-Fock (HF) or density functional theory (DFT), are not sufficient, but more reliable correlation methods are needed. Even low-level correlation methods like the second-order Møller-Plesset (MP2) perturbation theory ${ }^{1}$ are not accurate enough for most purposes, and the coupled-cluster (CC) approach ${ }^{2}$ is the method of choice if high-accuracy is required. Nevertheless, the CC model including single and double excitations (CCSD) ${ }^{3}$ is not significantly more trustworthy than modern DFT methods, and the consideration of triple excitations is also recommended ${ }^{4,5}$. The most successful CC approach including triple excitations is the CCSD with perturbative triples $[\operatorname{CCSD}(\mathrm{T})]$ method proposed by Raghavachari and coworkers ${ }^{6}$. The accuracy provided by this method is adequate for most chemical applications in the domain of single-reference systems, and it is often referred to as the "gold standard" of quantum chemistry. It had been originally derived for canonical HF references, but later it was also extended to the non-canonical case ${ }^{7,8}$. Subsequently, the analytic gradients ${ }^{9}$ and second-derivatives ${ }^{10-12}$ were also implemented for this method facilitating the computation of accurate properties. While its original proposition was more or less heuristic ${ }^{6}$, a more rigorous derivation of the $\operatorname{CCSD}(\mathrm{T})$ method was presented by Stanton ${ }^{13}$. His approach was later also the basis for the generalization of perturbative corrections to higher-order CC methods $^{14,15}$. Nowadays, several highly-efficient implementations of $\operatorname{CCSD}(\mathrm{T})$ facilitates its use $^{16-22}$, and the state-of-the-art local correlation techniques also enable $\operatorname{CCSD}(\mathrm{T})$ calculations for molecules of hundreds or thousands of atoms ${ }^{23-26}$.

Besides the level of correlation, the other factor seriously limiting the accuracy of computational results is the quality of the atomic orbital (AO) basis set. It has been known for a long time that large basis sets including functions of high angular momentum are required to reduce the error stemming from the incompleteness of the basis below an acceptable mag-

nitude. Though the complete basis set (CBS) limit can be sufficiently approached utilizing convergent basis set hierarchies ${ }^{27}$ and extrapolation techniques ${ }^{28-30}$, such calculations are still rather expensive with correlated methods.

A partial solution to this problem is offered by explicitly correlated approaches ${ }^{31-33}$. These approaches are based on wave functions that explicitly contain the interelectronic distances thereby significantly reducing the basis set requirements. The development of explicitly cor- 
related methods was initiated by Kutzelnigg in the mid 80 's ${ }^{34}$, and it was later continued by Kutzelnigg and Klopper ${ }^{31,35}$. In the early explicitly correlated models, termed the R12 ansätze, the correlation factor was linear in the interelectronic distance. The realization of this theory at the MP2 level was accomplished already in $1987^{35}$, and it was further improved in the 90 's ${ }^{31}$. Parallel to these efforts, the theory was also extended to the CCSD level by Noga et al. ${ }^{36}$ Over the years, the explicitly correlated theory went through significant evolution. The linear R12 correlation factor was replaced by the more advanced exponential F12 factors $^{37}$, which systematically outperform the linear factor. A fixed amplitude approach was proposed that remarkably simplifies the theory without sacrificing accuracy ${ }^{38}$. For the resolution of the identity approximation, which is the cornerstone of explicitly correlated methods, a new, numerically robust scheme, the complementary auxiliary basis (CABS) approach was introduced ${ }^{39,40}$. The density fitting (DF) technique, which considerably accelerates the evaluation of the required integrals, was adapted to explicitly correlated models ${ }^{41}$. Alternative formulations for the matrix elements of explicitly correlated MP2 theory were put forward that result in less demanding calculations and also improve the convergence properties toward the CBS limit ${ }^{42}$. Thanks to these achievements, by now MP2-F12 theory probably reached its final form ${ }^{43-45}$.

The CC theory also benefited from the aforementioned achievements. A fully explicitly correlated CCSD method with the Slater-type geminal correlation factor, CCSD-F12, was introduced and implemented ${ }^{46-48}$. Though this approach was more efficient than its predecessors, it was still too costly for general applications. At the same time, a more approximate explicitly correlated CCSD method, CCSD(F12), was also developed, which is almost as accurate as CCSD-F12 but only three to five times as expensive as standard CCSD ${ }^{49,50}$. Subsequently, even more cost-effective variants were proposed including the CCSD-F12a and CCSD-F12b schemes of Knizia, Adler, and Werner $(\mathrm{KAW})^{51,52}$, the CCSD $(2)_{\overline{\mathrm{F} 12}}$ approach of Valeev and Crawford ${ }^{53,54}$, and the $\operatorname{CCSD}\left(\mathrm{F} 12^{*}\right)$ method of Hättig and co-workers ${ }^{55}$. Benchmark studies show that these methods preserve the accuracy of the CCSD-F12 model, while their computational expenses are not more than 1.5-times higher than those for conventional $\mathrm{CCSD}^{56}$.

Concerning higher excitations, explicitly correlated iterative CC methods including up to quadruple excitations were published by Shiozaki and co-workers ${ }^{57}$. For the rigorous treatment of perturbative triple excitations, only one attempt has been made so far, by Köhn, 
who developed an explicitly correlated $(\mathrm{T})$ correction $^{58}$ based on his modified ansatz ${ }^{59}$. Unfortunately his approach is relatively expensive and turned out to be only moderately competitive with the conventional $(\mathrm{T})$ correction. A less rigorous approximation was proposed by $\mathrm{KAW}^{52}$, who simply scaled the $(\mathrm{T})$ correction computed in the conventional way by the ratio of the MP2-F12 and MP2 correlation energies. Though their scheme is practically costless and performs quite well in practical applications, it is unfortunately not size consistent, which may cause problems if the size of the system is increased. Here, we propose a similar heuristic approach, which is, however, size consistent.

\section{THEORY}

The $(\mathrm{T})$ correction can be evaluated $\mathrm{as}^{6-8}$

$$
E^{(\mathrm{T})}=\frac{1}{36} \sum_{i j k a b c} W_{i j k}^{a b c} t_{i j k}^{a b c}
$$

where $i, j, \ldots(a, b, \ldots)$ denote correlated (semi-)canonical spin-orbitals, while $p, q, \ldots$ will stand for generic indices. Here, $t_{i j k}^{a b c}$ is a second-order triple excitation amplitude expressed with converged CCSD doubles amplitudes, $t_{i j}^{a b}$, as

$$
t_{i j k}^{a b c}=\left[\hat{P}(a \mid b c) \hat{P}(i j \mid k) \sum_{d}\langle b c|| d k\rangle t_{i j}^{a d}-\hat{P}(a b \mid c) \hat{P}(i \mid j k) \sum_{l}\langle l c|| j k\rangle t_{i l}^{a b}\right] / D_{i j k}^{a b c},
$$

where $\langle p q \| r s\rangle$ is a two-electron integral in the Dirac notation, and $D_{i j k}^{a b c}$ is the usual energy denominator, $D_{i j \cdots}^{a b \cdots}=f_{i i}+f_{j j}+\cdots-f_{a a}-f_{b b}-\cdots$ with $f_{p q}$ as a Fock-matrix element. Operator $\hat{P}(p \mid q r)$ interchanges $p$ with indices $q$ and $r$ in all possible ways in the subsequent expression and multiplies it by $(-1)^{\mathcal{P}}$ with $\mathcal{P}$ being the parity of the permutation, e.g., $\hat{P}(a \mid b c) \sum_{d}\langle b c|| d k\rangle t_{i j}^{a d}=\sum_{d}\langle b c|| d k\rangle t_{i j}^{a d}-\sum_{d}\langle a c \| d k\rangle t_{i j}^{b d}+\sum_{d}\langle b a \| d k\rangle t_{i j}^{c d}$. Intermediate $W_{i j k}^{a b c}$ is defined by

$$
W_{i j k}^{a b c}=D_{i j k}^{a b c} t_{i j k}^{a b c}+\hat{P}(a b \mid c) \hat{P}(i \mid j k)\left(t_{i}^{a}\langle j k|| b c\rangle+f_{a i} t_{j k}^{b c}\right),
$$

where $t_{i}^{a}$ is a converged CCSD single excitation amplitude.

In most explicitly correlated $\operatorname{CCSD}(\mathrm{T})$ theories $^{50,51,55}$, the above perturbative triples correction is employed without any modification, and explicit correlation is only introduced at the CCSD level. Alternatively, one can use the pragmatic approach of $\mathrm{KAW}^{52}$ and scale the (T) correction as

$$
E^{(\mathrm{T} *)}=\frac{E^{\mathrm{MP} 2-\mathrm{F} 12}}{E^{\mathrm{MP} 2}} E^{(\mathrm{T})}
$$


Here, $E^{\mathrm{MP} 2}$ is the conventional MP2 correlation energy calculated with the given AO basis set as

$$
E^{\mathrm{MP} 2}=\sum_{a i} \frac{f_{a i}^{2}}{D_{i}^{a}}+\frac{1}{4} \sum_{i j a b} \frac{\langle a b \| i j\rangle^{2}}{D_{i j}^{a b}}
$$

whereas $E^{\mathrm{MP} 2-\mathrm{F} 12}$ denotes the MP2-F12 correlation energy,

$$
E^{\mathrm{MP} 2-\mathrm{F} 12}=E^{\mathrm{MP} 2}+E^{\mathrm{F} 12} .
$$

The expression for the F12 correction to the MP2 energy, $E^{\mathrm{F} 12}$, depends on the explicitly correlated approach. For instance, using the $2 \mathrm{~B}$ ansatz, the $\mathrm{F}+\mathrm{K}$ commutator approximation, and fixed amplitudes ${ }^{38,42,45}$, which approximations are utilized throughout the paper, the $E^{\mathrm{F} 12}$ contribution reads as

$$
E^{\mathrm{F} 12}=\frac{1}{2} \sum_{i j}\left(B_{i j}-X_{i j}+C_{i j}+V_{i j}\right)
$$

where intermediates $B, X, C$, and $V$ are introduced in Appendix A. The rationale behind this approach is that the MP2 correlation energy and the (T) correction scale similarly with the size of the basis set. This simple scheme performs remarkably well but unfortunately lacks size-consistency since the ratios $E^{\mathrm{MP} 2-\mathrm{F} 12} / E^{\mathrm{MP} 2}$ are, in general, different for different non-interacting subsystems ${ }^{52}$. A simple way to ensure size-consistency is to use the same scaling factor for all chemical species, determined, e.g., for the largest system ${ }^{23}$. However, this approach could be impractical when dealing with a large number of molecules exhibiting different rate of basis set convergence.

To define an improved $(\mathrm{T})$ correction, we follow a slightly different route. Instead of the entire MP2 correlation energy and (T) correction, we only suppose that the contributions of a particular molecular orbital (MO) to the these quantities scale similarly with the basis set size. Consequently, we scale the contribution of each MO to the (T) correction separately with the ratio of the corresponding second-order contributions. To that end, we rewrite the (T) correction by pulling out one of the occupied summation indices as

$$
E^{(\mathrm{T})}=\sum_{i} \frac{1}{36} \sum_{j k a b c} W_{i j k}^{a b c} t_{i j k}^{a b c}=\sum_{i} \delta E_{i}^{(\mathrm{T})} .
$$

Analogously, the MP2 correlation energy and its F12 correction can be recast as

$$
E^{\mathrm{MP} 2}=\sum_{i}\left(\sum_{a} \frac{f_{a i}^{2}}{D_{i}^{a}}+\frac{1}{4} \sum_{j a b} \frac{\langle a b|| i j\rangle^{2}}{D_{i j}^{a b}}\right)=\sum_{i} \delta E_{i}^{\mathrm{MP} 2}
$$


and

$$
E^{\mathrm{F} 12}=\sum_{i}\left(\frac{1}{2} \sum_{j} B_{i j}-X_{i j}+C_{i j}+V_{i j}\right)=\sum_{i} \delta E_{i}^{\mathrm{F} 12},
$$

respectively, and then the MP2-F12 correlation energy can also be split up as

$$
E^{\mathrm{MP} 2-\mathrm{F} 12}=\sum_{i}\left(\delta E_{i}^{\mathrm{MP} 2}+\delta E_{i}^{\mathrm{F} 12}\right)=\sum_{i} \delta E_{i}^{\mathrm{MP} 2-\mathrm{F} 12}
$$

With the above definitions, we are in the position to introduce our triple excitation correction, denoted as $(\mathrm{T}+)$, which reads as

$$
E^{(\mathrm{T}+)}=\sum_{i} \frac{\delta E_{i}^{\mathrm{MP} 2-\mathrm{F} 12}}{\delta E_{i}^{\mathrm{MP} 2}} \delta E_{i}^{(\mathrm{T})}=\sum_{i} q_{i} \delta E_{i}^{(\mathrm{T})} .
$$

Similar to the $\left(\mathrm{T}^{*}\right)$ approach, the $(\mathrm{T}+)$ correction can be combined with all flavors of explicitly correlated CCSD, and it also reduces to $(\mathrm{T})$ in the CBS limit because the $\delta E_{i}^{\mathrm{F} 12}$ terms vanish in that limit. On the other hand, since the contribution of an orbital is scaled separately with a factor that is specific to that orbital, the above correction is size-consistent (see Appendix B for an in depth discussion of size-consistency). Moreover, one also expects a more balanced performance from the $(\mathrm{T}+)$ correction as the treatment of the basis set incompleteness of the correlation contribution is orbital specific.

To further analyze the properties of the $(\mathrm{T}+)$ correction, we can rewrite it exploiting Eq. (8) and the antisymmetry of intermediate $W$ and the cluster amplitudes as

$$
\begin{aligned}
E^{(\mathrm{T}+)} & =\frac{1}{3}\left(\sum_{i} q_{i} \delta E_{i}^{(\mathrm{T})}+\sum_{j} q_{j} \delta E_{j}^{(\mathrm{T})}+\sum_{k} q_{k} \delta E_{k}^{(\mathrm{T})}\right) \\
& =\frac{1}{3}\left(\sum_{i} q_{i} \frac{1}{36} \sum_{j k a b c} W_{i j k}^{a b c} t_{i j k}^{a b c}+\sum_{j} q_{j} \frac{1}{36} \sum_{i k a b c} W_{j i k}^{a b c} t_{j i k}^{a b c}+\sum_{k} q_{k} \frac{1}{36} \sum_{j i a b c} W_{k j i}^{a b c} t_{k j i}^{a b c}\right) \\
& =\frac{1}{36} \sum_{i j k a b c} \frac{1}{3}\left(q_{i}+q_{j}+q_{k}\right) W_{i j k}^{a b c} t_{i j k}^{a b c} .
\end{aligned}
$$

First, the above equation offers an alternative interpretation of the $(\mathrm{T}+)$ correction. It suggests that $E^{(\mathrm{T}+)}$ can also be considered as a $(\mathrm{T})$ correction with a different scaling factor for each triplet of occupied orbitals. Second, Eq. (13) is more suitable for a computer implementation. As $q_{i}+q_{j}+q_{k}$ is symmetric, it can be easily implemented in existing $(\mathrm{T})$ codes irrespective of the order and restrictions of the loops running over the summation indices, and the additional costs arising due to the multiplication with the $q_{i}+q_{j}+q_{k}$ factors are negligible. Furthermore, the $\delta E_{i}^{\mathrm{MP} 2}$ and $\delta E_{i}^{\mathrm{F} 12}$ contributions required for the $q_{i}$ 
scaling factors can also be simply evaluated from quantities that are anyway computed in an MP2-F12 calculation preceding an explicitly correlated $\operatorname{CCSD}(\mathrm{T})$ calculation. Thus, the overhead of the $(\mathrm{T}+)$ correction is insignificant.

Eq. (13) also suggests that the contribution of the triplets of occupied orbitals could also be scaled with other quantities. An intuitive choice is to use the quotients of pair energies. The MP2, F12, and MP2-F12 pair energies are defined, respectively, as

$$
\begin{gathered}
\delta E_{i j}^{\mathrm{MP} 2}=\frac{1}{2} \sum_{a b} \frac{\langle a b \| i j\rangle^{2}}{D_{i j}^{a b}}, \\
\delta E_{i j}^{\mathrm{F} 12}=B_{i j}-X_{i j}+C_{i j}+V_{i j},
\end{gathered}
$$

and

$$
\delta E_{i j}^{\mathrm{MP} 2-\mathrm{F} 12}=\delta E_{i j}^{\mathrm{MP} 2}+\delta E_{i j}^{\mathrm{F} 12} .
$$

Note that, similar to the orbital contributions, the sum of the above pair energies over all pairs of orbitals gives back the total MP2 correlation energy, F12 correction, and MP2-F12 correlation energy, respectively. We can evaluate the ratios of the MP2-F12 and MP2 pair energies as

$$
q_{i j}=\frac{\delta E_{i j}^{\mathrm{MP} 2-\mathrm{F} 12}}{\delta E_{i j}^{\mathrm{MP} 2}},
$$

which quantities are proportional with the basis set incompleteness of the MP2 pair energies. Now, an alternative $(\mathrm{T}+)$ correction can be defined as

$$
E^{(\mathrm{T}+)^{\prime}}=\frac{1}{36} \sum_{i j k a b c} \frac{1}{3}\left(q_{i j}+q_{i k}+q_{j k}\right) W_{i j k}^{a b c} t_{i j k}^{a b c} .
$$

This triples correction has the same favorable properties as $(\mathrm{T}+)$, that is, it size-consistent, easy to implement, and practically costless. We also implemented this alternative form of the $(\mathrm{T}+)$ correction. In test calculations, we found that the performance of the two $(\mathrm{T}+)$ variants is similar, but in average, the first form of the $(\mathrm{T}+)$ correction is somewhat more accurate. Consequently, in the following, we will only discuss the first variant.

We note that here, our new approach has been introduced for the $(\mathrm{T})$ correction, but it can also be applied to reduce the basis set incompleteness error of other methods whose energy expression is similar to Eq. (1). These include numerous many-body methods, most notably, higher-order perturbative $\mathrm{CC}$ approaches, such as the $(\mathrm{Q})$ correction $^{14,15}$. These avenues will be explored in further studies. 


\section{BENCHMARK CALCULATIONS}

\section{A. Computational details}

The present approach has been implemented in the MRCC suite of quantum chemical programs $^{60,61}$, which is also used in all the calculations. The technical details of our explicitly correlated CCSD implementation will be discussed elsewhere. The conventional CCSD(T) parts of the computations utilize the hand-optimized, parallel and (partially) integral-direct open- and closed-shell DF-CCSD(T) implementations of the MrCC suite ${ }^{22,61,62}$.

As the AO basis set, the correlation consistent $X$-tuple- $\zeta$ cc-pVXZ-F12 $(X=\mathrm{D}$, $\mathrm{T}$, Q) sets developed for explicitly correlated calculations by Peterson et al. ${ }^{63}$ were employed together with the corresponding cc-pVXZ-F12-OPTRI CABS bases of Yousaf and Peterson $^{64,65}$. In all calculations, the DF approximation was invoked at both the $\mathrm{HF}$ and the correlated levels. In the explicitly correlated calculations, the fitting bases were the aug-cc$\mathrm{pV}(X+1)$ Z-RI-JK sets of Weigend ${ }^{66}$ and the aug-cc-pwCV $(X+1)$ Z-RI bases of Hättig ${ }^{67}$, respectively. The pseudopotential-based cc-pVXZ-PP-F12, cc-pVXZ-PP-F12-OPTRI, and cc-pVXZ-PP-F12-RI bases of Hill and Peterson ${ }^{68}$ were employed for the post-d main group elements in combination with the corresponding small-core pseudopotentials ${ }^{69,70}$. A Slatertype $f_{12}$ correlation factor was used with exponents of $0.9,1.0$, and 1.1 with the cc-pVDZF12, cc-pVTZ-F12, and cc-pVQZ-F12 basis sets, respectively ${ }^{63}$. Six Gaussians were used to fit the correlation factor, and the expansion coefficients were taken from Ref. 71. In the reference calculations with the conventional $\operatorname{CCSD}(\mathrm{T})$ method, Dunning's augmented correlation consistent pentuple- and hextuple- $\zeta$ basis $\operatorname{sets}^{27,72-74}$ were used together with the corresponding $\mathrm{HF}^{66}$ and correlation ${ }^{75}$ fitting bases.

For the CCSD step of the explicitly correlated CC calculations, the CCSD(F12*) approach of Hättig and co-workers was applied ${ }^{55}$. For the open-shell species, restricted open-shell HF orbitals were used. The core electrons were frozen in all correlation calculations.

For benchmarking our method for thermochemistry, the closed- and open-shell reaction energy and atomization energy test sets of $\mathrm{KAW}^{52}$ were taken. Only those species were considered for which canonical $\operatorname{CCSD}(\mathrm{T})$ reference results extrapolated from pentuple- and hextuple- $\zeta$ basis sets were published in Ref. 52 , but the reference values were recalculated to avoid any inconsistency. The test sets include 28 and 48 reactions of closed- and open-shell 
systems, respectively, as well as 49 atomization energies.

The performance of the new method for molecular properties was assessed for the test set of Knizia $^{76}$ including six closed-shell ( $\mathrm{HF}, \mathrm{N}_{2}, \mathrm{CO}, \mathrm{BF}, \mathrm{F}_{2}, \mathrm{C}_{2}$ ) and seven open-shell $\left(\mathrm{OH}, \mathrm{NH}, \mathrm{CH}, \mathrm{CN}, \mathrm{NO}, \mathrm{O}_{2}, \mathrm{CF}\right)$ diatomic molecules. The bond lengths and the harmonic vibrational frequencies were computed for the lowest electronic state of the species. The geometry optimizations were carried out with the Newton method applying numerical derivatives. The force constants were evaluated via the 5-point central difference formula. The reference values were also recomputed.

Further test calculations were carried out for the complexes of the water molecule and the chloride anion with $\pi$-electron systems. The complexes of water with ethyne, benzene, and pyridine were taken from the S66 compilation of Řezáč et al. ${ }^{77}$ From the anion- $\pi$ test set of Garau and co-workers ${ }^{78}$, the complexes of the chloride ion with $s$-triazine (TAZ) and trifluoro-s-triazine (TFZ) were considered. A linked system of two TAZ moieties was also studied (L-TAZ 2 of Fig. 1) representing realistic systems designed for selective halidebinding ${ }^{79}$. Small halogen-bonded complexes of hydrogen cyanide and formaldehyde with nine dihalogen molecules were taken from the XB18 compilation of Kozuch and Martin ${ }^{80}$.

To characterize the performance of the methods, the mean absolute error (MAE), the root mean square (RMS) deviation, and the maximum error (MAX) of the computed results will be applied.

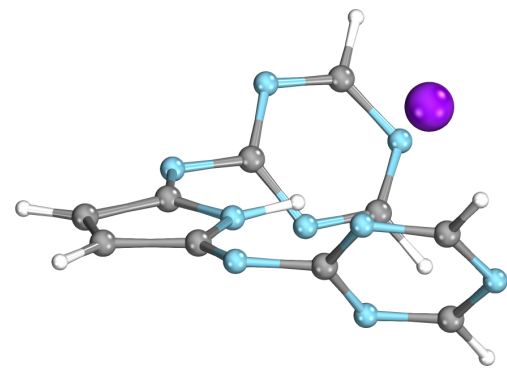

FIG. 1. A linked system of two $s$-triazine moieties $\left(\mathrm{L}-\mathrm{TAZ}_{2}\right)$ binding with an iodide ion as a representative of realistic halide-binding ion traps ${ }^{79}$. 


\section{B. Atomization energies}

To evaluate the performance of new method, first, the atomization energies were computed for the species of the KAW test set. The statistical error measures for the $(\mathrm{T}),\left(\mathrm{T}^{*}\right)$, and $(\mathrm{T}+)$ corrections, all computed with converged $\mathrm{CCSD}\left(\mathrm{F} 12^{*}\right)$ amplitudes, are compiled in Table I.

TABLE I. Errors of perturbative triples corrections (in $\mathrm{kJ} / \mathrm{mol}$ ) for the atomization energies of the KAW test set.

\begin{tabular}{ccccc}
\hline \hline Basis set & Error & $(\mathrm{T})$ & $\left(\mathrm{T}^{*}\right)$ & $(\mathrm{T}+)$ \\
\hline cc-pVDZ-F12 & MAE & 4.03 & 0.84 & 0.83 \\
& RMS & 4.77 & 1.18 & 1.15 \\
& MAX & 10.09 & 3.00 & 2.95 \\
cc-pVTZ-F12 & MAE & 1.82 & 0.26 & 0.24 \\
& RMS & 2.16 & 0.34 & 0.32 \\
& MAX & 4.82 & 0.88 & 0.74 \\
cc-pVQZ-F12 & MAE & 0.88 & 0.14 & 0.12 \\
& RMS & 1.06 & 0.18 & 0.16 \\
& MAX & 2.30 & 0.56 & 0.46 \\
\hline \hline
\end{tabular}

As we can see, the performance of the $(\mathrm{T}+)$ correction is very similar to that of $\left(\mathrm{T}^{*}\right)$. Both methods effectively reduce the basis-set error of the (T) contribution. Depending on the basis set, they decrease the average (maximum) error by a factor of 4 to 7 (3 to 6 ). The gain in the accuracy is significantly larger with the triple- $\zeta$ basis set than with cc-pVDZ-F12, and it is only moderately smaller with the quadruple- $\zeta$ basis. Of course, we should keep in mind that the uncertainty of the reference data is almost as large as the errors of the $\left(\mathrm{T}^{*}\right)$ and $(\mathrm{T}+)$ approaches, thus the cc-pVQZ-F12 results are less representative. Concerning the basis set convergence, the error is reduced by about a factor of 3 to 4 when going from cc-pVDZ-F12 to cc-pVTZ-F12, while the improvement is more moderate when the cardinal number of the basis set is increased to 4 . Nevertheless, the errors are under $1 \mathrm{~kJ} / \mathrm{mol}$ already with the triple- $\zeta$ basis set. Consequently, this basis set can be used if an error of 1 $\mathrm{kJ} / \mathrm{mol}$ is acceptable. The results suggest that $(\mathrm{T}+)$ slightly but consistently outperforms 
$\left(\mathrm{T}^{*}\right)$. Again, for the aforementioned reason, only the differences of about $0.1 \mathrm{~kJ} / \mathrm{mol}$ in the maximum errors can be considered significant from this point of view as the differences of the MAE and RMS values are smaller than the uncertainty of the reference.

\section{Reaction energies}

Next, we analyze the errors of the reaction energies obtained for the test set of KAW. The results for the reactions of closed- and open-shell species are presented in Tables II and III, respectively.

TABLE II. Errors of perturbative triples corrections (in $\mathrm{kJ} / \mathrm{mol}$ ) for the closed-shell reaction energies of the KAW test set.

\begin{tabular}{ccccc}
\hline \hline Basis set & Error & $(\mathrm{T})$ & $\left(\mathrm{T}^{*}\right)$ & $(\mathrm{T}+)$ \\
\hline cc-pVDZ-F12 & MAE & 1.75 & 1.06 & 1.04 \\
& RMS & 2.81 & 1.42 & 1.40 \\
& MAX & 9.21 & 3.48 & 3.35 \\
cc-pVTZ-F12 & MAE & 0.77 & 0.38 & 0.39 \\
& RMS & 1.36 & 0.55 & 0.55 \\
& MAX & 5.18 & 1.90 & 1.84 \\
cc-pVQZ-F12 & MAE & 0.37 & 0.14 & 0.15 \\
& RMS & 0.65 & 0.19 & 0.20 \\
& MAX & 2.43 & 0.48 & 0.46 \\
\hline \hline
\end{tabular}

Inspecting the error metrics for the reactions involving only closed-shell molecules, we can see that the behavior of the various triples corrections is somewhat different from that observed for the atomization energies. While the error of the canonical $(\mathrm{T})$ correction is smaller for the reaction energies than for the atomization energies, the improvement brought by the scaled variants is smaller (cf. Tables I and II), especially with the triple- $\zeta$ basis. They only decrease the average (maximum) error by a factor of 2 to 3 (2 to 5 ), and these factors are larger for the quadruple- $\zeta$ basis set. The basis set convergence is also slower: the basis set error is halved between the double- and triple- $\zeta$ bases, but the reduction is larger when going to cc-pVQZ-F12. Whereas the average error is smaller than $1 \mathrm{~kJ} / \mathrm{mol}$ with cc-pVTZ- 
F12, the quadruple- $\zeta$ basis is needed to achieve this accuracy in the maximum errors. The performances of the $\left(\mathrm{T}^{*}\right)$ and $(\mathrm{T}+)$ schemes are also very similar in this case. The average errors are indistinguishable, while the maximum errors are slightly better for $(\mathrm{T}+)$ with the cc-pVDZ-F12 and cc-pVTZ-F12 basis sets.

TABLE III. Errors of perturbative triples corrections (in $\mathrm{kJ} / \mathrm{mol}$ ) for the open-shell reaction energies of the KAW test set.

\begin{tabular}{ccccc}
\hline \hline Basis set & Error & $(\mathrm{T})$ & $\left(\mathrm{T}^{*}\right)$ & $(\mathrm{T}+)$ \\
\hline cc-pVDZ-F12 & MAE & 2.72 & 1.28 & 1.29 \\
& RMS & 3.30 & 1.88 & 1.90 \\
& MAX & 10.01 & 6.97 & 6.93 \\
cc-pVTZ-F12 & MAE & 1.19 & 0.55 & 0.55 \\
& RMS & 1.48 & 0.84 & 0.83 \\
& MAX & 4.07 & 2.65 & 2.56 \\
cc-pVQZ-F12 & MAE & 0.58 & 0.28 & 0.27 \\
& RMS & 0.72 & 0.45 & 0.44 \\
& MAX & 1.83 & 1.47 & 1.46 \\
\hline \hline
\end{tabular}

The conclusions are similar for the reactions containing also open-shell species, but the improvement over the canonical $(\mathrm{T})$ correction is even more moderate. The scaled $(\mathrm{T})$ variants reduce the average basis set error by a factor of about 2 independently of the basis set, and the drop in the maximum error is even smaller. The basis set convergence is also slow, the basis set error is only reduced by a factor of 2 when increasing the cardinal number by 1 . Seeking the reason for the less satisfactory performance of the modified (T) corrections for the open-shell reactions, one finds that their error is relatively large for particular species, e.g., the oxygen molecule. These species appear in a couple of reactions with big stoichiometric coefficients resulting in large errors for those reactions and worsening the error statistics. The average performances of the $\left(\mathrm{T}^{*}\right)$ and $(\mathrm{T}+)$ approaches is again indistinguishable. A tiny statistically significant difference can only be observed for the maximum error with the triple- $\zeta$ basis set. 


\section{Molecular properties}

To also evaluate the performance of the approximate triple excitation models for molecular properties, the bond lengths and harmonic vibrational frequencies were calculated for the test set of Knizia ${ }^{76}$. The error measures for the bond lengths and frequencies are presented in Tables IV and V, respectively.

TABLE IV. Errors of perturbative triples contributions (in pm) to the bond lengths of diatomic molecules.

\begin{tabular}{ccccc}
\hline \hline Basis set & Error & $(\mathrm{T})$ & $\left(\mathrm{T}^{*}\right)$ & $(\mathrm{T}+)$ \\
\hline cc-pVDZ-F12 & MAE & 0.108 & 0.021 & 0.028 \\
& RMS & 0.147 & 0.024 & 0.043 \\
& MAX & 0.424 & 0.049 & 0.131 \\
cc-pVTZ-F12 & MAE & 0.042 & 0.008 & 0.009 \\
& RMS & 0.057 & 0.009 & 0.012 \\
& MAX & 0.160 & 0.015 & 0.028 \\
cc-pVQZ-F12 & MAE & 0.022 & 0.004 & 0.005 \\
& RMS & 0.031 & 0.005 & 0.007 \\
& MAX & 0.091 & 0.010 & 0.019 \\
\hline \hline
\end{tabular}

Concerning bond lengths, the conclusions are in line with those for the thermochemical properties, but here, the $\left(\mathrm{T}^{*}\right)$ approach seems to perform somewhat better than $(\mathrm{T}+)$, especially the maximum deviations are considerably better with the former. The $\left(\mathrm{T}^{*}\right)$ approach reduces the average (maximum) error of the genuine $(\mathrm{T})$ method by factors of 5 to 7 (9 to 11) with the various basis sets, while the corresponding values vary between 3 and 5 (3 and 6) with $(\mathrm{T}+)$. As to the basis set convergence, we realize that the error is decreased by about a factor of 3 (2) when going from cc-pVDZ-F12 to cc-pVTZ-F12 (cc-pVTZ-F12 to cc-pVQZ-F12). The average accuracy of $0.0001 \AA$ achieved with the triple- $\zeta$ basis is already acceptable for almost all applications.

Turning our attention to the frequencies, we can see that, for this property, the improvement over the conventional $(\mathrm{T})$ approach is less pronounced. The scaled corrections reduce the error of $(\mathrm{T})$ by a factor of $3-4$, and the performance of $\left(\mathrm{T}^{*}\right)$ is again somewhat more 
TABLE V. Errors of perturbative triples contributions $\left(\right.$ in $\mathrm{cm}^{-1}$ ) to harmonic vibrational frequencies of diatomic molecules.

\begin{tabular}{ccccc}
\hline \hline Basis set & Error & $(\mathrm{T})$ & $\left(\mathrm{T}^{*}\right)$ & $(\mathrm{T}+)$ \\
\hline cc-pVDZ-F12 & MAE & 8.5 & 2.4 & 3.0 \\
& RMS & 9.5 & 2.9 & 3.7 \\
& MAX & 19.1 & 5.3 & 7.7 \\
cc-pVTZ-F12 & MAE & 3.4 & 0.8 & 1.0 \\
& RMS & 3.8 & 1.0 & 1.2 \\
& MAX & 7.4 & 1.8 & 2.3 \\
cc-pVQZ-F12 & MAE & 1.7 & 0.4 & 0.5 \\
& RMS & 1.9 & 0.6 & 0.7 \\
& MAX & 3.8 & 1.2 & 1.5 \\
\hline \hline
\end{tabular}

convincing. The basis set convergence follows a similar pattern as that of the bond lengths: the accuracy is improved by factors of about 3 and 2 when increasing the cardinal number from 2 to 3 and 3 to 4 , respectively. The accuracy attained with the cc-pVTZ-F12 basis is also quite high and sufficient for most purposes, but spectroscopic, i.e., sub-cm ${ }^{-1}$ accuracy cannot be achieved even with the quadruple- $\zeta$ basis set. Of course, we should not forget that the remaining basis set error of the reference is still about $0.1-0.2 \mathrm{~cm}^{-1}$, thus the reference does not approach spectroscopic accuracy either.

Though the major focus of this paper is on the perturbative triple excitation corrections, it is also instructive to inspect the overall performance of the $\operatorname{CCSD}\left(\mathrm{F} 12^{*}\right)(\mathrm{T})$, $\operatorname{CCSD}\left(\mathrm{F} 12^{*}\right)\left(\mathrm{T}^{*}\right)$, and $\operatorname{CCSD}\left(\mathrm{F} 12^{*}\right)(\mathrm{T}+)$ methods (see the Supplementary material). As the basis set error of $\operatorname{CCSD}\left(\mathrm{F} 12^{*}\right)$ may cancel with the error of $(\mathrm{T})$, it might happen that $\operatorname{CCSD}\left(\mathrm{F} 12^{*}\right)(\mathrm{T})$ is better than the more advanced approaches. Fortunately, this only occurs for the bond lengths with the cc-pVDZ-F12 basis. Otherwise, the modified CCSD $\left(\mathrm{F} 12^{*}\right)(\mathrm{T})$ models are superior to $\operatorname{CCSD}\left(\mathrm{F} 12^{*}\right)(\mathrm{T})$, and the performance of $\operatorname{CCSD}\left(\mathrm{F} 12^{*}\right)\left(\mathrm{T}^{*}\right)$ and $\mathrm{CCSD}\left(\mathrm{F} 12^{*}\right)(\mathrm{T}+)$ is almost indistinguishable. 


\section{E. Binding energies of complexes}

As it has been revealed above, the $(\mathrm{T}+)$ correction is quite accurate but its performance is similar to that of the $\left(\mathrm{T}^{*}\right)$ approach, probably because the size-consistency error (SCE) is not pronounced for the small systems considered hitherto. Big SCEs can be expected for processes where systems of different size and electronic structure interact. Thus, to demonstrate the benefits of the $(\mathrm{T}+)$ correction, first, we carried out benchmark calculations for the complexes of the water molecule and the chloride anion with $\pi$-electron systems. We computed the (unrelaxed) complexation energies with the various perturbative triples approaches as well as the SCE of the $\left(\mathrm{T}^{*}\right)$ correction. The latter quantity was calculated as the energy of the supersystem separated by a sufficiently large distance minus the energies of the aromatic molecule and the other species. The results are presented in Table VI.

In absolute terms, the difference between the $\left(\mathrm{T}^{*}\right)$ and $(\mathrm{T}+)$ approaches and the SCE of the former are not large. Obviously, the correlation between these quantities is perfect, which means that the SCE directly propagates into the $\left(\mathrm{T}^{*}\right)$ binding energies. The SCE, and thus the difference of $\left(\mathrm{T}^{*}\right)$ and $(\mathrm{T}+)$ reaches its maximum in the cc-pVDZ-F12 basis and diminishes with increasing basis set size as the F12 contribution and consequently the SCE goes to zero in the CBS limit. The largest absolute error, $2.5 \mathrm{~kJ} / \mathrm{mol}$, is measured for the $\mathrm{L}_{-} \mathrm{TAZ}_{2}$-iodide complex with the double- $\zeta$ basis. The errors are also above $1 \mathrm{~kJ} / \mathrm{mol}$ for the complexes of TAZ and TFZ with chloride and about $0.4-0.5 \mathrm{~kJ} / \mathrm{mol}$ for the water complexes. With the triple- $\zeta$ basis set, the SCE can still be as large as $0.4 \mathrm{~kJ} / \mathrm{mol}$, and it drops to about $0.1 \mathrm{~kJ} / \mathrm{mol}$ with cc-pVQZ-F12. The SCE obviously slows down the basis set convergence of the $\left(\mathrm{T}^{*}\right)$ correction. While $(\mathrm{T}+)$ converges to about $0.1 \mathrm{~kJ} / \mathrm{mol}$ in the triple- $\zeta$ basis, the basis set error of the $\left(\mathrm{T}^{*}\right)$ correction is so large even with the quadruple- $\zeta$ basis. It is also important to realize that, in most cases, the error of the $\left(\mathrm{T}^{*}\right)$ approach is larger than that of the genuine $(\mathrm{T})$ correction.

Even though the absolute SCEs of the $\left(\mathrm{T}^{*}\right)$ method seem acceptable, its relative errors are pronounced. They fluctuate in the 20-50\% interval with the double- $\zeta$ basis set and can still exceed $20 \%$ using the cc-pVTZ-F12 basis. Of course, similar relative errors are expected for more extensive systems, that is, the absolute errors can be much larger.

An alternative source of SCE could be the different rate of basis set convergence within the subsystems. In such cases, the scaling factors of $\left(\mathrm{T}^{*}\right)$ are expected to differ markedly 
TABLE VI. Perturbative triples corrections and SCEs for the $\left(\mathrm{T}^{*}\right)$ approach (in $\mathrm{kJ} / \mathrm{mol}$ ) for the binding energies of weakly-bound complexes. All signs are reversed.

\begin{tabular}{|c|c|c|c|c|c|}
\hline Complex & Basis & $(\mathrm{T})$ & $\left(\mathrm{T}^{*}\right)$ & $(\mathrm{T}+)$ & SCE \\
\hline \multirow[t]{3}{*}{ Ethyne $\cdots \mathrm{H}_{2} \mathrm{O}$} & cc-pVDZ-F12 & 0.59 & 1.06 & 0.70 & 0.38 \\
\hline & cc-pVTZ-F12 & 0.59 & 0.80 & 0.63 & 0.17 \\
\hline & cc-pVQZ-F12 & 0.61 & 0.71 & 0.63 & 0.08 \\
\hline \multirow[t]{3}{*}{ Benzene $\cdots \mathrm{H}_{2} \mathrm{O}$} & cc-pVDZ-F12 & 1.64 & 2.35 & 1.92 & 0.48 \\
\hline & cc-pVTZ-F12 & 1.83 & 2.18 & 1.94 & 0.27 \\
\hline & cc-pVQZ-F12 & 1.85 & 2.00 & 1.89 & 0.12 \\
\hline \multirow[t]{3}{*}{ Pyridine $\cdots \mathrm{H}_{2} \mathrm{O}$} & cc-pVDZ-F12 & 1.80 & 2.49 & 2.07 & 0.46 \\
\hline & cc-pVTZ-F12 & 1.87 & 2.22 & 1.99 & 0.27 \\
\hline & cc-pVQZ-F12 & 1.88 & 2.04 & 1.93 & 0.12 \\
\hline \multirow[t]{3}{*}{$\mathrm{TAZ} \cdot \mathrm{Cl}^{-}$} & cc-pVDZ-F12 & 3.24 & 5.52 & 4.00 & 1.73 \\
\hline & cc-pVTZ-F12 & 3.44 & 4.05 & 3.78 & 0.37 \\
\hline & cc-pVQZ-F12 & 3.55 & 3.83 & 3.70 & 0.17 \\
\hline \multirow[t]{3}{*}{ TFZ $\cdots \mathrm{Cl}^{-}$} & cc-pVDZ-F12 & 3.92 & 5.73 & 5.02 & 1.02 \\
\hline & cc-pVTZ-F12 & 4.14 & 4.54 & 4.56 & 0.06 \\
\hline & cc-pVQZ-F12 & 4.32 & 4.46 & 4.50 & 0.00 \\
\hline $\mathrm{L}^{-\mathrm{TAZ}_{2}} \cdots \mathrm{I}^{-}$ & cc-pVDZ-F12 & 11.0 & 15.0 & 13.9 & 2.51 \\
\hline
\end{tabular}

for the monomers and the complex. The halogen-bonded dimers of the XB18 compilation appear to be particularly SCE sensitive from this perspective (see Table VII). The largest SCE, $1.7 \mathrm{~kJ} / \mathrm{mol}$, with cc-pVDZ-F12 was found for the iodine-hydrogen cyanide complex. While the average SCE of $\left(\mathrm{T}^{*}\right)$ for the nine $\mathrm{HCN}$-dihalogen complexes of the XB18 set is $1.3 \mathrm{~kJ} / \mathrm{mol}$ with the cc-pVDZ-F12 basis set, the analogous average SCE for the other nine, formaldehyde-dihalogen type complexes is only $0.5 \mathrm{~kJ} / \mathrm{mol}$. On the example of $\mathrm{HCN} \cdots \mathrm{I}_{2}$ in Table VII, we again find a rapid decrease of SCE with increasing basis sets: the SCE drops 
to 0.2 and $0.02 \mathrm{~kJ} / \mathrm{mol}$ with the cc-pVTZ-F12 and cc-pVQZ-F12 bases, respectively.

TABLE VII. Perturbative triples corrections and SCEs for the $\left(\mathrm{T}^{*}\right)$ approach (in $\mathrm{kJ} / \mathrm{mol}$ ) for the binding energies of SCE-sensitive complexes. All signs are reversed.

\begin{tabular}{cccccc}
\hline \hline Complex & Basis & $(\mathrm{T})$ & $\left(\mathrm{T}^{*}\right)$ & $(\mathrm{T}+)$ & $\mathrm{SCE}$ \\
\hline HCN $\cdots \mathrm{I}_{2}$ & cc-pVDZ-F12 & 1.64 & 3.72 & 2.16 & 1.68 \\
& cc-pVTZ-F12 & 2.10 & 2.50 & 2.29 & 0.22 \\
& cc-pVQZ-F12 & 2.22 & 2.33 & 2.31 & 0.02 \\
$(\mathrm{CN})_{2} \cdots \mathrm{I}_{2}$ & cc-pVDZ-F12 & 4.08 & 9.49 & 5.72 & 4.47 \\
& cc-pVTZ-F12 & 4.97 & 6.46 & 5.66 & 1.01 \\
& cc-pVQZ-F12 & 5.50 & 6.03 & 5.80 & 0.30 \\
$\mathrm{HCN} \cdots \mathrm{Xe}_{2}$ & cc-pVDZ-F12 & 0.31 & 4.23 & 0.43 & 3.82 \\
& cc-pVTZ-F12 & 0.51 & 1.84 & 0.59 & 1.27 \\
& cc-pVQZ-F12 & 0.57 & 1.03 & 0.61 & 0.43 \\
\hline \hline
\end{tabular}

The extensivity of the SCE in $\left(\mathrm{T}^{*}\right)$ is illustrated for the complex of cyanogen with iodine molecules $\left[(\mathrm{CN})_{2} \cdots 2 \mathrm{I}_{2}\right.$ of Table VII]. Compared to the similar $\mathrm{HCN} \cdots \mathrm{I}_{2}$ complex, a difference of more than twice as large is found between the $\left(\mathrm{T}^{*}\right)$ and $(\mathrm{T}+)$ results, while the corresponding relative errors remain comparable (66-72\% with cc-pVDZ-F12, which drops to 9-14\% with cc-pVTZ-F12). In rare and particularly unfortunate cases, e.g., when the iodine atoms of $\mathrm{HCN} \cdots \mathrm{I}_{2}$ are replaced with xenon atoms, the combination of the small interaction energies and the sizeable SCE could lead to large relative errors, which remain $214 \%$ and $69 \%$ even with triple- and quadruple- $\zeta$ basis sets, respectively (see Table VII). The unscaled $(\mathrm{T})$ and the $(\mathrm{T}+)$ binding energy components converge to the same value also for the complexes of Table VII. Again, the rate of convergence is accelerated for the $(\mathrm{T}+)$ approach: the basis set incompleteness of $(\mathrm{T}+)$ appears to be below $0.2 \mathrm{~kJ} / \mathrm{mol}$ already with cc-pVDZ-F12.

These findings point out that the $\left(\mathrm{T}^{*}\right)$ correction should be employed with caution for larger systems, especially with smaller basis sets or with subsystems including markedly different atom types. Since the $(\mathrm{T}+)$ approach is free from the SCE, its performance is 
expected to be stable for bigger systems.

\section{CONCLUSIONS}

A new approach has been presented to approximate the CBS limit of the (T) correction in explicitly correlated CCSD $(\mathrm{T})$ calculations. Just as the $\left(\mathrm{T}^{*}\right)$ correction of Knizia et al..$^{52}$, our $(\mathrm{T}+)$ approach utilizes the ratio of MP2-F12 and MP2 correlation contributions. However, in our scheme, the correlation energy is broken down into orbital contributions, which are then scaled separately ensuring the size-consistency of our method. The new approach can simply be implemented in existing $(\mathrm{T})$ codes, and the additional costs incurring for the evaluation of the $(\mathrm{T}+)$ correction are negligible.

The new method has been benchmarked for atomization and reaction energies of smaller systems. Our results show that the $(\mathrm{T}+)$ correction efficiently decreases the basis-set incompleteness error of the perturbative triple excitation correction. Depending on the system and the basis set, the error can be reduced by factors of 2 to 7 . In further test calculations for bigger systems, we have demonstrated the advantages of the size-consistency of our approach. Our results also reveal that the size-consistency error of the $\left(\mathrm{T}^{*}\right)$ correction can be sizeable for larger systems, which can be problematic, for example, when the approach is used together with explicitly correlated local CCSD methods.

The basic idea of the $(\mathrm{T}+)$ approach, that is, the separate scaling of the correlation contributions of the orbitals can also be used to cure the basis-set incompleteness of other correlation methods. This will be the subject of future investigations.

\section{SUPPLEMENTARY MATERIAL}

See the supplementary material for the calculated correlation, atomization, and reaction energies; binding energies of the complexes; bond lengths and vibrational frequencies; and geometries of the model systems that are not available in the literature.

\section{ACKNOWLEDGMENTS}

The valuable discussions with Professor David P. Tew are gratefully acknowledged. The authors are indebted to Professor Gerald Knizia for his helpful comments on the manuscript. 
The authors are grateful for the financial support from the National Research, Development, and Innovation Office (NKFIH, Grant No. KKP126451). The research reported in this paper and carried out at BME has been supported by the NRDI Fund (TKP2020 IES, Grant No. BME-IE-BIO) based on the charter of bolster issued by the NRDI Office under the auspices of the Ministry for Innovation and Technology. The work of PRN is supported by the UNKP20-5 New National Excellence Program of the Ministry for Innovation and Technology from the source of the National Research, Development and Innovation Fund and the János Bolyai Research Scholarship of the Hungarian Academy of Sciences. The computing time granted on the Hungarian HPC Infrastructure at NIIF Institute, Hungary, and the DECI resource Saga based in Norway at Trondheim with support from the PRACE aisbl are gratefully acknowledged.

\section{DATA AVAILABILITY}

The data that supports the findings of this study are available within the article and its supplementary material.

\section{Appendix A: MP2-F12 intermediates}

Here, we define the intermediates for $E^{\mathrm{F} 12}$ supposing ansatz $2 \mathrm{~B}$, the $\mathrm{F}+\mathrm{K}$ commutator approximation, and fixed amplitudes ${ }^{38,42,45}$. 


$$
\begin{aligned}
& B_{i j}=\left\langle i j\left|\hat{S}_{i j}^{+}\left(\hat{\nabla}_{1} f_{12}\right)^{2} \hat{S}_{i j}\right| i j\right\rangle \\
& +\left\langle i j\left|\hat{S}_{i j}^{+} f_{12}^{2} \hat{S}_{i j}\right| \tilde{i} j\right\rangle+\left\langle i j\left|\hat{S}_{i j}^{+} f_{12}^{2} \hat{S}_{i j}\right| i \tilde{j}\right\rangle \\
& +\sum_{p^{\prime} q^{\prime} s^{\prime}}\left\langle p^{\prime} q^{\prime}\left|f_{12} \hat{S}_{i j}\right| i j\right\rangle k_{p^{\prime} s^{\prime}}\left\langle s^{\prime} q^{\prime}\left|f_{12} \hat{S}_{i j}\right| i j\right\rangle \\
& -\sum_{p^{\prime} m s^{\prime}}\left\langle p^{\prime} m\left|f_{12} \hat{S}_{i j}\right| i j\right\rangle f_{p^{\prime} s^{\prime}}\left\langle s^{\prime} m\left|f_{12} \hat{S}_{i j}\right| i j\right\rangle \\
& +\sum_{m a^{\prime} n}\left\langle m a^{\prime}\left|f_{12} \hat{S}_{i j}\right| i j\right\rangle f_{m n}\left\langle n a^{\prime}\left|f_{12} \hat{S}_{i j}\right| i j\right\rangle \\
& -\sum_{p a s}\left\langle p a\left|f_{12} \hat{S}_{i j}\right| i j\right\rangle f_{p s}\left\langle s a\left|f_{12} \hat{S}_{i j}\right| i j\right\rangle \\
& -2 \sum_{m a^{\prime} s^{\prime}}\left\langle m a^{\prime}\left|f_{12} \hat{S}_{i j}\right| i j\right\rangle f_{m s^{\prime}}\left\langle s^{\prime} a^{\prime}\left|f_{12} \hat{S}_{i j}\right| i j\right\rangle \\
& -2 \sum_{p a a^{\prime}}\left\langle p a\left|f_{12} \hat{S}_{i j}\right| i j\right\rangle f_{p a^{\prime}}\left\langle a^{\prime} a\left|f_{12} \hat{S}_{i j}\right| i j\right\rangle \\
& X_{i j}=\hat{P}_{+}(i \mid j) \sum_{k} f_{k j}\left(\left\langle i j\left|\hat{S}_{i j}^{+} f_{12}^{2} \hat{S}_{i k}\right| i k\right\rangle\right. \\
& +\sum_{p<q}\left\langle p q\left|f_{12} \hat{S}_{i j}\right| i j\right\rangle\left\langle p q\left|f_{12} \hat{S}_{i k}\right| i k\right\rangle \\
& \left.+\sum_{m a^{\prime}}\left\langle a^{\prime} m\left|f_{12} \hat{S}_{i j}\right| i j\right\rangle\left\langle a^{\prime} m\left|f_{12} \hat{S}_{i k}\right| i k\right\rangle\right) \\
& C_{i j}=\left[\sum_{a<b}\left(2\langle a b \| i j\rangle+R_{i j}^{a b}\right) R_{i j}^{a b}\right] / D_{i j}^{a b} \\
& V_{i j}=2\left(\left\langle i j\left|f_{12} r_{12}^{-1} \hat{S}_{i j}\right| i j\right\rangle\right. \\
& -\sum_{p<q}\left\langle p q\left|f_{12} \hat{S}_{i j}\right| i j\right\rangle\langle p q \| i j\rangle \\
& \left.-\sum_{m a^{\prime}}\left\langle a^{\prime} m\left|f_{12} \hat{S}_{i j}\right| i j\right\rangle\left\langle a^{\prime} m \| i j\right\rangle\right)
\end{aligned}
$$

In the above equations, $f_{12}$ is the correlation factor, $|i j\rangle$ stands for a Slater determinant composed of orbitals $i$ and $j$, and $\hat{\nabla}_{1}$ denotes the del operator with respect to the coordinates of the first electron. $p^{\prime}$ and $q^{\prime}$ are orbitals in the MO plus CABS basis, $a^{\prime}$ and $b^{\prime}$ denote complementary virtual orbitals represented in the CABS basis, while indices $m$ and $n$ run 
over the frozen core and correlated occupied orbitals. The rational generator $\hat{S}_{i j}$ is defined as

$$
\hat{S}_{i j}=\frac{3}{8}+\frac{1}{8} \hat{P}_{i j}
$$

where $\hat{P}_{i j}$ permutes the spatial components of spin orbitals $i$ and $j$ in determinant $|i j\rangle$. Indices with tilde refer to orbitals transformed by the Fock-matrix where the exchange terms are neglected, that is,

$$
|\tilde{i}\rangle=\sum_{p^{\prime}}\left(f_{i p^{\prime}}+k_{i p^{\prime}}\right)\left|p^{\prime}\right\rangle
$$

where $k_{i p^{\prime}}$ is an element of the exchange matrix. Operator $\hat{P}_{+}(i \mid j)$ has the same effect as $\hat{P}(i \mid j)$ but does not change the sign of the terms. $r_{12}$ is the distance of the two electrons. Finally, intermediate $R$ is evaluated as

$$
R_{i j}^{a b}=\hat{P}(a \mid b) \sum_{a^{\prime}} f_{a a^{\prime}}\left\langle a^{\prime} b\left|f_{12} \hat{S}_{i j}\right| i j\right\rangle .
$$

\section{Appendix B: Size-consistency of the $(\mathrm{T}+)$ ansatz}

The $(\mathrm{T}+)$ ansatz is trivially size-consistent if the occupied orbitals are localized on the non-interacting subsystems. This is usually the case for canonical HF orbitals. Exceptions are the systems where two or more orbitals of different subsystems are degenerate and can therefore mix with each other. This may happen for identical subsystems arranged symmetrically, or if the orbitals of the subsystems are accidentally degenerate.

To address this problem, let us consider two different subsystems with two degenerate orbitals, $i$ and $j$. In the subspace spanned by the latter, there always exit two orbitals, $i^{\prime}$ and $j^{\prime}$, which are localized on the subsystems. Then, the two pairs of orbitals can be transformed into each other by a unitary rotation parameterized by angle $\phi$ as

$$
\begin{aligned}
& |i\rangle=\cos \phi\left|i^{\prime}\right\rangle-\sin \phi\left|j^{\prime}\right\rangle \\
& |j\rangle=\sin \phi\left|i^{\prime}\right\rangle+\cos \phi\left|j^{\prime}\right\rangle .
\end{aligned}
$$

Utilizing that the energy denominators are invariant to this transformation, and that the $\delta E_{i}^{\mathrm{MP} 2}$ contributions are also invariant to the unitary transformation of the orbitals, one can simply show that

$$
\begin{aligned}
& \delta E_{i}^{\mathrm{MP} 2}=\cos ^{2} \phi \delta E_{i^{\prime}}^{\mathrm{MP} 2}+\sin ^{2} \phi \delta E_{j^{\prime}}^{\mathrm{MP} 2} \\
& \delta E_{j}^{\mathrm{MP} 2}=\sin ^{2} \phi \delta E_{i^{\prime}}^{\mathrm{MP} 2}+\cos ^{2} \phi \delta E_{j^{\prime}}^{\mathrm{MP} 2},
\end{aligned}
$$


and similar expressions hold for the $\delta E_{i}^{\mathrm{MP} 2-\mathrm{F} 12}$ and $\delta E_{i}^{(\mathrm{T})}$ increments. For symmetrically arranged identical subsystems, $\delta E_{i^{\prime}}^{\mathrm{MP} 2}$ and $\delta E_{j^{\prime}}^{\mathrm{MP} 2}$ are equal, and consequently the $\delta E_{i}^{\mathrm{MP} 2}$ contributions are invariant to the rotations of the degenerate orbitals. Since the same is true for $\delta E_{i}^{\mathrm{MP} 2-\mathrm{F} 12}$ and $\delta E_{i}^{(\mathrm{T})}$, the $(\mathrm{T}+)$ correction is also insensitive to these orbital mixings.

Unfortunately, in the unlikely event the two orbitals are accidentally degenerate, the $\delta E_{i^{\prime}}$ and $\delta E_{j^{\prime}}$ contributions are not identical, and the $(\mathrm{T}+)$ correction is not invariant to their rotation. As it rarely happens, this inconsistency is mainly important only from the theoretical point of view. Nevertheless, we were curious how large the size-consistency error is in such situations. To that end, using the cc-pVDZ-F12 basis set, we assembled a model system with accidentally degenerate orbitals. Our system consisted of an $\mathrm{Ar}$ and two $\mathrm{Ne}$ atoms placed in an L-shaped position. The Ar atom and the first Ne was located $10000 \AA$ from each other, while the distance between the first $\mathrm{Ne}$ and the additional Ne atom was adjusted to make the $3 \mathrm{~s}$ orbital of the Ar and an MO formed from the $2 \mathrm{~s}$ orbitals of the Ne's degenerate. We could not achieve such a high level of numerical degeneracy that the diagonalizer itself could automatically mix those orbitals, but we simply rotated the orbitals according to Eq. (B1). We found that the error did not exceed $4 \mu \mathrm{E}_{h}$ for any $\phi$, which is negligible

As it is also obvious from the above analysis, the $(\mathrm{T}+)$ correction is not invariant to the unitary rotation of the MOs. It does not raise any issue if $(\mathrm{T}+)$ is employed on top of canonical explicitly correlated CCSD methods. It may be, of course, problematic for local CC methods, where localized MOs are used. However, in many cases, the perturbative triples correction is evaluated in a semicanonical MO basis, which eliminates this problem. The application of the $(\mathrm{T}+)$ correction in local correlation methods will be discussed in subsequent publications.

\section{REFERENCES}

${ }^{1}$ C. Møller and M. S. Plesset, Phys. Rev. 46, 618 (1934).

${ }^{2}$ J. Č́žek, J. Chem. Phys. 45, 4256 (1966).

${ }^{3}$ G. D. Purvis III and R. J. Bartlett, J. Chem. Phys. 76, 1910 (1982).

${ }^{4}$ A. Tajti, P. G. Szalay, A. G. Császár, M. Kállay, J. Gauss, E. F. Valeev, B. A. Flowers, J. Vázquez and J. F. Stanton, J. Chem. Phys. 121, 11599 (2004). 
${ }^{5}$ A. Karton, E. Rabinovich, J. M. L. Martin and B. Ruscic, J. Chem. Phys. 125, 144108 (2006).

${ }^{6}$ K. Raghavachari, G. W. Trucks, J. A. Pople and M. Head-Gordon, Chem. Phys. Lett. 157, 479 (1989).

${ }^{7}$ J. D. Watts, J. Gauss and R. J. Bartlett, J. Chem. Phys. 98, 8718 (1993).

${ }^{8}$ M. J. O. Deegan and P. J. Knowles, Chem. Phys. Lett. 227, 321 (1994).

${ }^{9}$ J. D. Watts, J. Gauss and R. J. Bartlett, Chem. Phys. Lett. 200, 1 (1992).

${ }^{10}$ J. Gauss and J. F. Stanton, Chem. Phys. Lett. 276, 70 (1997).

${ }^{11}$ J. Gauss and J. F. Stanton, J. Chem. Phys. 104, 2574 (1996).

${ }^{12}$ P. G. Szalay, J. Gauss and J. F. Stanton, Theor. Chem. Acc. 100, 5 (1998).

${ }^{13}$ J. F. Stanton, Chem. Phys. Lett. 281, 130 (1997).

${ }^{14}$ Y. J. Bomble, J. F. Stanton, M. Kállay and J. Gauss, J. Chem. Phys. 123, 054101 (2005).

${ }^{15}$ M. Kállay and J. Gauss, J. Chem. Phys. 123, 214105 (2005).

${ }^{16}$ T. Janowski and P. Pulay, J. Chem. Theory Comput. 4, 1585 (2008).

${ }^{17}$ M. Pitoňák, F. Aquilante, P. Hobza, P. Neogrády, J. Noga and M. Urban, Collect. Czech. Chem. Commun. 76, 713 (2011).

${ }^{18}$ A. E. DePrince and C. D. Sherrill, J. Chem. Theory Comput. 9, 2687 (2013).

${ }^{19}$ A. Asadchev and M. S. Gordon, J. Chem. Theory Comput. 9, 3385 (2013).

${ }^{20}$ V. M. Anisimov, G. H. Bauer, K. Chadalavada, R. M. Olson, J. W. Glenski, W. T. C. Kramer, E. Aprà and K. Kowalski, J. Chem. Theory Comput. 10, 4307 (2014).

${ }^{21}$ C. Peng, J. A. Calvin and E. F. Valeev, Int. J. Quantum Chem. 119, e25894 (2019).

${ }^{22}$ L. Gyevi-Nagy, M. Kállay and P. R. Nagy, J. Chem. Theory Comput. 16, 366 (2020).

${ }^{23}$ Q. Ma and H.-J. Werner, Wiley Interdiscip. Rev.: Comput. Mol. Sci. 8, e1371 (2018).

${ }^{24}$ Y. Guo, C. Riplinger, U. Becker, D. G. Liakos, Y. Minenkov, L. Cavallo and F. Neese, J. Chem. Phys. 148, 011101 (2018).

${ }^{25}$ G. Schmitz, C. Hättig and D. P. Tew, Phys. Chem. Chem. Phys. 16, 22167 (2014).

${ }^{26}$ P. R. Nagy, G. Samu and M. Kállay, J. Chem. Theory Comput. 14, 4193 (2018).

${ }^{27}$ T. H. Dunning Jr., J. Chem. Phys. 90, 1007 (1989).

${ }^{28}$ T. Helgaker, W. Klopper, H. Koch and J. Noga, J. Chem. Phys. 106, 9639 (1997).

${ }^{29}$ K. D. Vogiatzis, E. C. Barnes and W. Klopper, Chem. Phys. Lett. 503, 157 (2011).

${ }^{30}$ D. S. Ranasinghe and G. A. Petersson, J. Chem. Phys. 138, 144104 (2013).

${ }^{31}$ W. Kutzelnigg and W. Klopper, J. Chem. Phys. 94, 1985 (1991). 
${ }^{32}$ W. Klopper, F. R. Manby, S.Ten-no and E. F. Valeev, Int. Rev. Phys. Chem. 25, 427 (2006).

${ }^{33}$ C. Hättig, W. Klopper, A. Köhn and D. P. Tew, Chem. Rev. 112, 4 (2012).

${ }^{34}$ W. Kutzelnigg, Theor. Chem. Acc. 68, 445 (1985).

${ }^{35}$ W. Klopper and W. Kutzelnigg, Chem. Phys. Lett. 134, 17 (1987).

${ }^{36}$ J. Noga, W. Kutzelnigg and W. Klopper, Chem. Phys. Lett. 199, 497 (1992).

${ }^{37}$ S. Ten-no, Chem. Phys. Lett. 398, 117 (2004).

${ }^{38}$ S. Ten-no, J. Chem. Phys. 121, 117 (2004).

${ }^{39}$ W. Klopper and C. C. M. Samson, J. Chem. Phys. 116, 6397 (2002).

${ }^{40}$ E. F. Valeev, Chem. Phys. Lett. 395, 190 (2004).

${ }^{41}$ F. R. Manby, J. Chem. Phys. 119, 4607 (2003).

${ }^{42}$ S. Kedžuch, M. Milko and J. Noga, Int. J. Quantum Chem. 105, 929 (2005).

${ }^{43}$ H.-J. Werner, T. B. Adler and F. R. Manby, J. Chem. Phys. 126, 164102 (2007).

${ }^{44}$ G. Knizia and H.-J. Werner, J. Chem. Phys. 128, 154103 (2008).

${ }^{45}$ R. A. Bachorz, F. A. Bischoff, A. Glöss, C. Hättig, S. Höfener, W. Klopper and D. P. Tew, J. Comput. Chem. 32, 2492 (2011).

${ }^{46}$ J. Noga, S. Kedžuch, J. Šimunek and S. Ten-no, J. Chem. Phys. 128, 174103 (2008).

${ }^{47}$ T. Shiozaki, M. Kamiya, S. Hirata and E. F. Valeev, J. Chem. Phys. 129, 071101 (2008).

${ }^{48}$ A. Köhn, G. W. Richings and D. P. Tew, J. Chem. Phys. 129, 201103 (2008).

${ }^{49}$ H. Fliegl, W. Klopper and C. Hättig, J. Chem. Phys. 122, 084107 (2005).

${ }^{50}$ D. P. Tew, W. Klopper, C. Neiss and C. Hättig, Phys. Chem. Chem. Phys. 9, 1921 (2007).

${ }^{51}$ T. B. Adler, G. Knizia and H.-J. Werner, J. Chem. Phys. 127, 221106 (2007).

${ }^{52}$ G. Knizia, T. B. Adler and H.-J. Werner, J. Chem. Phys. 130, 054104 (2009).

${ }^{53}$ M. Torheyden and E. F. Valeev, Phys. Chem. Chem. Phys. 10, 3410 (2008).

${ }^{54}$ E. F. Valeev and T. Daniel Crawford, J. Chem. Phys. 128, 244113 (2008).

${ }^{55}$ C. Hättig, D. P. Tew and A. Köhn, J. Chem. Phys. 132, 231102 (2010).

${ }^{56}$ M. K. Kesharwani, N. Sylvetsky, A. Köhn, D. P. Tew and J. M. L. Martin, J. Chem. Phys. 149, 154109 (2018).

${ }^{57}$ T. Shiozaki, M. Kamiya, S. Hirata and E. F. Valeev, J. Chem. Phys. 130, 054101 (2009).

${ }^{58}$ A. Köhn, J. Chem. Phys. 130, 131101 (2009).

${ }^{59}$ A. Köhn, J. Chem. Phys. 130, 104104 (2009).

${ }^{60}$ M. Kállay, P. R. Nagy, D. Mester, Z. Rolik, G. Samu, J. Csontos, J. Csóka, P. B. Szabó, 
L. Gyevi-Nagy, B. Hégely, I. Ladjánszki, L. Szegedy, B. Ladóczki, K. Petrov, M. Farkas,

P. D. Mezei, Á. Ganyecz, and R. A. Horváth, Mrcc, a quantum chemical program suite. See https://www.mrcc.hu/. (Accessed June 1, 2021).

${ }^{61}$ M. Kállay, P. R. Nagy, D. Mester, Z. Rolik, G. Samu, J. Csontos, J. Csóka, P. B. Szabó, L. Gyevi-Nagy, B. Hégely, I. Ladjánszki, L. Szegedy, B. Ladóczki, K. Petrov, M. Farkas, P. D. Mezei, and Á. Ganyecz, J. Chem. Phys. 152, 074107 (2020).

${ }^{62}$ L. Gyevi-Nagy, M. Kállay and P. R. Nagy, J. Chem. Theory Comput. 17, 860 (2021).

${ }^{63}$ K. A. Peterson, T. B. Adler and H.-J. Werner, J. Chem. Phys. 128, 084102 (2008).

${ }^{64}$ K. E. Yousaf and K. A. Peterson, J. Chem. Phys. 129 (2008).

${ }^{65}$ K. E. Yousaf and K. A. Peterson, Chem. Phys. Lett. 476 (2009).

${ }^{66}$ F. Weigend, J. Comput. Chem. 29, 167 (2008).

${ }^{67}$ C. Hättig, Phys. Chem. Chem. Phys. 7, 59 (2005).

${ }^{68}$ J. G. Hill and K. A. Peterson, J. Chem. Phys. 141, 094106 (2014).

${ }^{69}$ K. A. Peterson, B. C. Shepler, D. Figgen and H. Stoll, J. Phys. Chem. A. 110, 13877 (2006).

${ }^{70}$ K. A. Peterson, D. Figgen, E. Goll, H. Stoll and M. Dolg, J. Chem. Phys. 119, 11113 (2003).

${ }^{71}$ D. P. Tew and W. Klopper, J. Chem. Phys. 123, 074101 (2005).

${ }^{72}$ R. A. Kendall, T. H. Dunning Jr. and R. J. Harrison, J. Chem. Phys. 96, 6796 (1992).

${ }^{73}$ T. van Mourik, A. K. Wilson and T. H. Dunning, Jr, Mol. Phys. 96, 529 (1999).

${ }^{74}$ A. K. Wilson, T. van Mourik and T. H. Dunning, J. Mol. Struct. (Theochem). 388, 339 (1996).

${ }^{75}$ F. Weigend, A. Köhn and C. Hättig, J. Chem. Phys. 116, 3175 (2002).

${ }^{76}$ G. Knizia. Explicitly correlated quantum chemistry methods for high-spin open-shell molecules. PhD thesis, Institut für Theoretische Chemie der Universität Stuttgart, 2010.

${ }^{77}$ J. Řezáč, K. E. Riley and P. Hobza, J. Chem. Theory Comput. 7, 2427 (2011).

${ }^{78}$ C. Garau, A. Frontera, D. Quiñonero, N. Russo and P. M. Deyà, J. Chem. Theory Comput. 7, $3012(2011)$.

${ }^{79}$ H. Wang, W. Wang and W. J. Jin, Chem. Rev. 116, 5072 (2016).

${ }^{80}$ S. Kozuch and J. M. L. Martin, J. Chem. Theory Comput. 9, 1918 (2013). 